\title{
Experimental study of the bouncing trajectory of a particle along a rotating wall
}

\author{
A.Le Quiniou (1), F.Rioual (1), P. Héritier (1) and Y. Lapusta (2) \\ (1)Cemagref Clermont-Ferrand (Montoldre sur Allier) \\ Domaine des Palaquins, \\ 03150, Montoldre, France \\ (2)Laboratoire LAMI, Institut Français de Mécanique Avancée(IFMA) \\ Campus des Cézeaux, \\ BP265, 63175 Aubière, France,
}

\begin{abstract}
We intend to present a new experimental setup that allows the study of the trajectory of a solid spherical particle bouncing at a high velocity along a rotating plate. Using different surface treatments for the plate, we can explore the phase space for the mechanical parameters of the problem (normal restitution coefficient $e_{n}$ and dynamic friction coefficient $\mu$ ). An accurate statistical analysis of the trajectory (radial and angular velocities) has been conducted based on an image analysis procedure. Experiments show a regime of successive bounces, followed by a regime of permanent contact of the particle along the vane and a transition from a rolling with sliding regime to a rolling without sliding regime triggered by the friction particle/wall. A simple model using two mechanical parameters (normal coefficient of restitution $e_{n}$ and friction coefficient $\mu$ ) as proposed recently [A.Le Quiniou and F.Rioual, EPL 8234001 (2008)] is sufficient to reproduce quantitatively all the features of the trajectory. The friction coefficient has to be determined independently using a mechanical protocol of impact of a single particle on a fixed wall - following [S.F.Foerster, M.Y.Louge, H.Chang, and K.Allia, Phys.Fluids 6, 1108 (1994)] - in particular, an outcome of this study is that the initial spin of the particle appears to have no effect on the features of the impact as long as the relative velocities at the contact are considered.
\end{abstract}

\section{Introduction}

Impacts of particles on boundaries are still the object of important investigations from material scientists. They represent indeed the basic ingredients to take into account for modelling particulate flows along boundaries. The challenge is to be able to describe these flows in a simple way, using macroscopic mechanical parameters, the energy an momentum exchanges between the colliding objects during the impact. Hence the introduction of a coefficient of restitution in order to describe this energy exchange. Starting from the analysis of Hertz-Mindlin and the analytical theory of Maw [1], the Walton model [2] is classically used in order to describe the features of the impact. This is a three parameter model :

- two parameters which describe the behaviour of the center of mass : the normal restitution coefficient $e_{n}$ and the friction coefficient $\mu$,

- and one parameter which represents the tangential motion at the contact patch produced by the tangential compliance : the tangential restitution coefficient $\beta$. Maw [1] and Johnson [3] have indeed demonstrated the need to consider tangential elastic compliance over the contact area. This model has received several criticisms, in particular for the determination of the tangential coefficient of restitution. This parameter is supposed to take into account the tangential compliance. For nearly head-on impacts, the tangential compliance induces normally a possible reversal of the tangential component of the relative velocity at contact and this is a typical situation where the value of this tangential coefficient becomes inexact [4]. The effect of the initial spin on the impact has often been omitted in previous experimental studies. This might not be insignificant because collisional flows demonstrate frequently oblique impacts between particles and with boundaries which generate some spin. Recently, a study of the impact of particles with initial spins has been conducted [5]. The authors argued that it remains a challenge to model the event of elastic impacts with initial spins. C.Thornton [6] proposed recently that these aspects of the rebound kinematics can be normalized as soon as we use an effective impact angle, defined as the ratio of the relative tangential to normal surface velocities of the two impacting bodies. The study of the bouncing motion of a particle on a static wall has also been studied in other contexts [7] where the finite number of bounces results from a trade-off between gravity and visco-elastic dissipation at the wall. Recently, the impact of a particle on a rotating plate has been studied experimentally in the case where the particle falls vertically under its own weight. Experiments demonstrated the possibility of rolling without sliding for the particle all along the impact [8].

Here we present a new experimental setup devoted to the study of the trajectory of spinning and bouncing particles along a rotating boundary. The particles considered here are plastic model spheres which are used in air guns (Polyvinylchloride). This setup is inspired partly by machines used for spreading fertilizers in which a quantitative prediction of the flow induced the inertial forces (centrifugal, coriolis) for general granules is not an obvious issue [9]. In the first part of this letter, we present the experimental setup and the experimental procedure used for the study of the trajectories. In the second one, we recall the main features of the model that has been 
proposed recently for a description of the trajectory. The third part focuses on the experimental caracterisation of the frictional interaction between a particle and the boundary and in particular the determination of the mechanical parameters involved in the model(restitution coefficient $e_{n}$, friction coefficient $\mu$ ). In the fourth part, we discuss the features of the trajectory and interpret the experimental results at the light of the model. We show in particular that we are able to predict quantitatively the value of the critical friction coefficient $\mu^{\star}$ for which rolling without sliding appears along the trajectory of the particle.

\section{EXPERIMENTAL SETUP}

A schematic set-up of the experiment is shown in figure (Fig. 1).

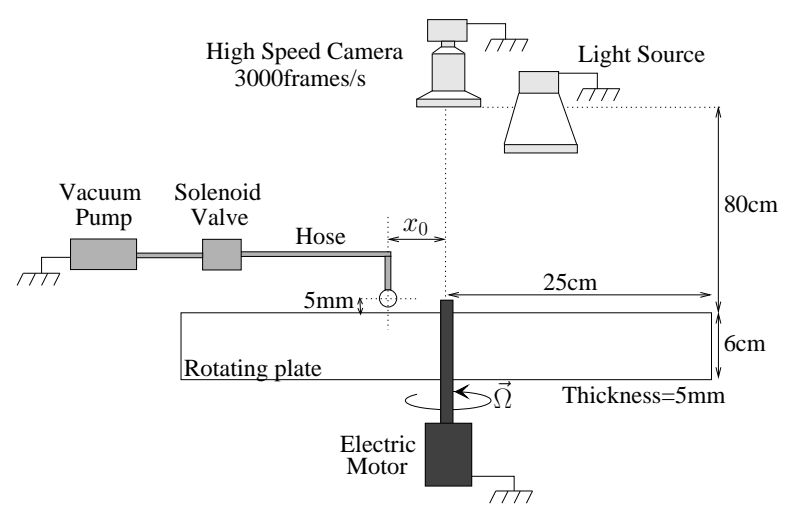

FIGURE 1: Schematic experimental set-up

The particles considered in these experiments are $B B$ beads of diameter $5.93 \pm 0.02$, the density is $1.86 \pm$ $0.01\left(\mathrm{~g} / \mathrm{cm}^{3}\right)$ and the mass $\mathrm{m}$ is $0.21 \mathrm{~g}$. The mechanical properties of the particles have been determined from experiments of binary collisions between these particles [10] and give $: e_{n}=0.91, \mu=0.19$ and $E(\mathrm{~Pa})=2.8 \cdot 10^{9}$. In these experiments, the particle is held to a hose by a vacuum created with a vacuum pump (the solenoid valve is opened). In order to release the particle, an electronic device generates a sequence of pulses (not shown in the picture) in order to trigger the camera and to activate the solenoid valve which closes the valve and stops the vacuum. The particle falls without spinning, under gravity, onto the thick rotating plane from a constant minimal height $(\approx 5 \mathrm{~mm}$.) in order to avoid any height effects [8]. A digital video camera with spot illumination is used to record the trajectory of the particle at a resolution of $(1024 * 1024)$ pixels and a rate of $f=3000$ frames per second. A $1 \mathrm{~mm}$ wide white band is drawn on the equator of spherical particles (with a black background) in order to indicate their orientations and to deduce their angular velocities. Figure 2 shows a typical sequence of the trajectory for a model particle along a rotating plate and Figure 3 shows a typical sequence of its angular velocity (in the fixed frame of the laboratory). These sequences of frames are further analysed using image processing sofware (ImageJ). The measurements of the particle positions and angles give us informations on the radial and rotational velocities and indirectly other quantities like the normal restitution coefficient and the dynamical friction coefficient.

The random errors on the particle position on the rotating plate are estimated as $\delta x= \pm 2 \mathrm{~mm}$ (errors on particle and plate positions). The random errors on the model particle angles are $\delta \theta= \pm 5^{\circ}$. At the end of the plate (ejected radial position $x_{e}=25 \mathrm{~cm}$ ), the random errors on the radial velocity are an estimated $\frac{\delta V_{x}}{V_{x}}=\frac{\delta x}{x_{t+1}-x_{t}}=1 \%$ and on the rotational velocity $\frac{R \delta \dot{\theta}}{R \dot{\theta}}=\frac{\delta \theta}{\theta_{t+1}-\theta_{t}}=3 \%$. Statistics based on fifteen samples have been conducted for each configuration (angular velocity of the plate $\Omega \in[500-1000] \mathrm{rpm}$, initial radial position $x_{0} \in[1-8]$ $\mathrm{cm}$, variation of the plate material). The normality of the population distribution has been tested by a $Q-Q$ plot.

Sondergaard et al 1990 [11] showed that the normal restitution coefficient decreases with the ratio of sphere diameter to plate thickness in agreement with the theory of Zener [12]. In this study, we use a plate with a sufficient thickness $(5 \mathrm{~mm}$.) in order to avoid any reduction of the restitution coefficient related to this effect. Five different surface treatments on the plates have been undertaken in order to modify the characteristics of the interaction and thus to explore the mechanical phase space (coefficient of restitution $e_{n}$, friction coefficient $\mu$ ). (I) A stainless steel plate painted black (technical painting Julien noir), (II) A steel plate sand-blasted (sand-blasting), (III) A plate varnished (Vernish astral wood), (IV) A plate polished (with emery paper, P80), (V) A plate in steel which has been rubber coated.

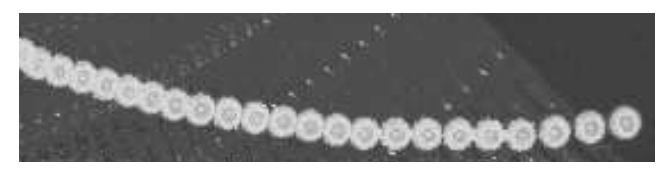

FIGURE 2: Typical sequence showing the spinning motion of a spherical particle onto the rotating plate.

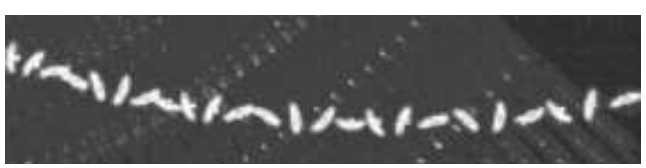

Figure 3: Typical sequence showing the angular velocity of a spherical particle onto the rotating plate. 


\section{OVERVIEW OF THE BASIC ASSUMPTIONS OF THE THEORY FOR THE PARTICLE'S TRAJECTORY}

An analytical treatment of the trajectory of a particle along the rotating vane carried out recently [13]. The trajectory of a spherical particle (radius $R$, mass $m$ and normal contact stiffness $K_{n}$ and dissipation parameter $\nu)$ at an initial radial position $x_{0}$ in the rotating frame $\left(R^{\prime}\right)$, consists of a succession of bounces followed by a permanent contact of the particle along the plate (see Fig. 4). The theoretical description used is based on the rigid body theory introducing only two mechanical parameters for the impact : a coefficient of restitution $e_{n}$ and a friction coefficient $\mu$. This model is applicable if the dimensionless collision time $\left(N_{t}=\Omega t_{c}\right)$ and the centrifugal number $\left(N_{c}=\frac{x_{0} \Omega}{R / t_{c}}\right)$ are small compared to one i.e. the collision is considered as instantaneous and as a collision on a static plate $\left(t_{c}\right.$ is the collision time on a static plate). This is a valid assumption for most rigid materials. The model predicts in particular the existence of a critical friction coefficient $\mu^{\star}$ between the particle and the plate for which the particle can roll without any sliding in the sticking phase. This critical friction coefficient appears to depend only on the normal restitution coefficient $e_{n}$ of the particle and $\mu^{\star}=\frac{1}{\sqrt{35}}(\approx 0.169)$ is a good approximation according to the theory at least for high normal restitution coefficients $\left(e_{n}>0.3\right)$.

The experimental setup presented here can test precisely these different issues for the trajectory of a spinning particle along a rotating boundary.

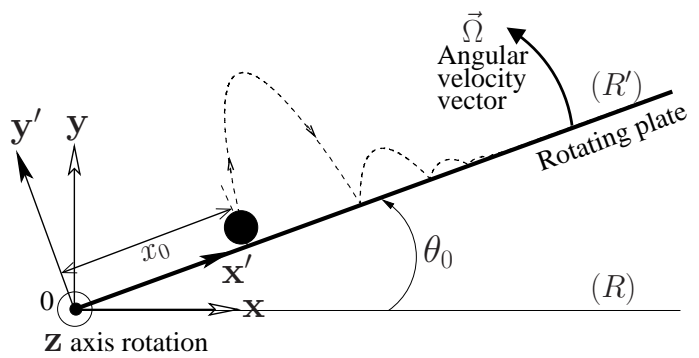

FiguRE 4: Schematic view of the trajectory of a spherical particle along the rotating plate in the rotating frame.

\section{INTERACTION BETWEEN A MOVING BEAD AND A BOUNDARY}

Determining experimentally the mechanical quantities which are relevant for the characterization of the impact in the problem is not a simple task and in particular the value of the friction coefficient (particle/wall).

Firstly, we measured these parameters independantly based on an experiment of collision of a falling bead on a static plate : to determine these three constant parameters, we use the model of Foerster et al 1994 [10] which is a simplification of the model of Maw et al 1981 [1] in the definition of the impact and rebound angles with spherical particles. In this setup, the particle falls from a constant height $(h \approx 25 \mathrm{~cm})$ without initial spin under gravity onto an inclined plate from $\gamma=0^{\circ}$ to $80^{\circ}$ which corresponds to an impact velocity of $V_{i}=\sqrt{2 g h}=2.21$ $\mathrm{m} / \mathrm{s}$.

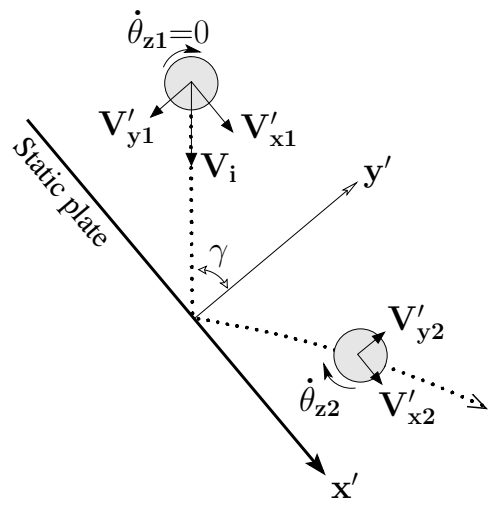

FIGURE 5: Schematic impact of a sphere on an oblique static plate $\gamma$. Substripts 1 and 2 refer to initial values and those after rebound, respectively.

The velocities before and after impact are decomposed into their normal and tangential components at the impact point (see Fig. 5). The velocity before impact obtained from video analysis is $\left(V_{x 1}^{\prime}, V_{y 1}^{\prime}, \dot{\theta}_{z 1}=0\right)$ and the final velocity is $\left(V_{x 2}^{\prime}, V_{y 2}^{\prime}, \dot{\theta}_{z 2}\right)$. Starting from the rigid body theory, the absence of initial rotational speed gives the expression of the rotational speed for a sphere after impact equal to :

$$
R \dot{\theta}_{z 2}=\frac{5}{2}\left(V_{x 2}^{\prime}-V_{x 1}^{\prime}\right)
$$

The quantity $\dot{\theta}_{z 2}$ has been measured directly and also calculated indirectly using equation 1 . We find a reasonable agreement between the two sets of data, see figure Fig. 6 . The fact that the rotational speed calculated from Eq. 1 is based on the assumption that deformations are small indicates that the rigid body theory is still valid. Gorham and Kharaz [14] have also drawn the same conclusions about collisions with plastic deformations (aluminium oxide spheres on an aluminium plate).

Following [10] and varying the inclination of the plate, it is shown that a three parameter model (normal coefficient of restitution $e_{n}$, tangential coefficient of restitution $\beta$, friction coefficient $\mu$ ) can capture the features of the impact. We define the relative translational velocity at the contact patch before and after impact : $g_{x 1,2} \equiv V_{x 1,2}^{\prime}-R \dot{\theta}_{z 1,2}$. Foerster et al propose a nondimensional local angle of contact before and after an impact :

$$
\psi_{1,2}=\frac{g_{x 1,2}}{V_{y 1}^{\prime}}
$$




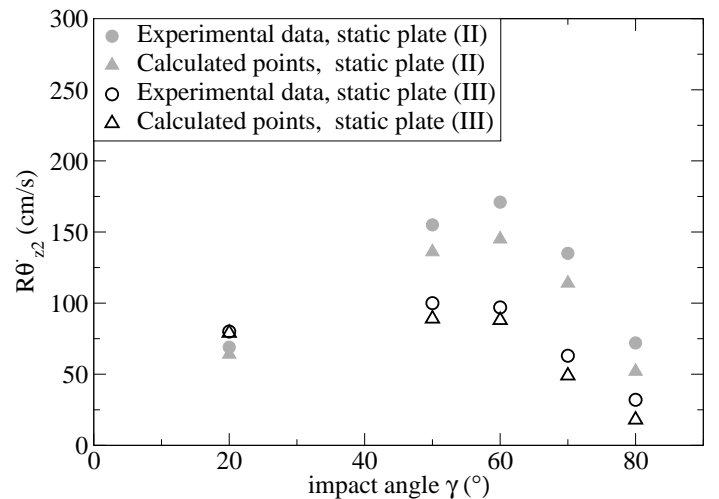

FiguRE 6: Rotational speed directly measured and rotational speed calculated from 1 for model particles on surfaces (I) and surfaces (II).

Walton's model implies that slipping and sticking regions do not coexist in a given impact. The application of this model leads to a sliding solution of the form :

$$
\psi_{2}=\psi_{1}-\frac{7}{2}\left(1+e_{n}\right) \mu
$$

For non-sliding collisions (sticking), the obtained solution is :

$$
\psi_{2}=-\beta \psi_{1}
$$

From the model we can represent the collision diagram i.e. plot the non-dimensional local angle of contact after an impact $\psi_{2}$ as a function of the non-dimensional local angle of contact before an impact $\psi_{1}$. From such a plot, the parameters involved in eqs. (3) and (4) can be used to determine $\mu$ and $\beta$, respectively.

The experimental plot of $\psi_{2}$ as a function of $\psi_{1}$ is shown on Fig. 7 for the impact of the plastic particle onto the five different plates. In each case, the two parameters $\mu$ and $\beta$ have been determined.

The values of the impact parameters $e_{n}$ and $\mu$ for the five different plates are summarised in the Table I.

TABLE I: Summary of the mechanical parameters for the collision of the model particles with the fixed plate.

\begin{tabular}{ccc}
\hline \hline Plate material & $e_{n}$ & $\mu$ \\
\hline plate (I) & $0.875 \pm 0.02$ & $0.175 \pm 0.005$ \\
plate (II) & $0.76 \pm 0.06$ & $0.45 \pm 0.01$ \\
plate (III) & $0.68 \pm 0.06$ & $0.164 \pm 0.005$ \\
plate (IV) & $0.88 \pm 0.05$ & $0.205 \pm 0.003$ \\
plate (V) & $0.80 \pm 0.06$ & $0.64 \pm 0.01$ \\
\hline \hline
\end{tabular}

The next figure represents $\Psi_{2}$ as a function of $\Psi_{1}$ for both experimental configurations (the static plate and

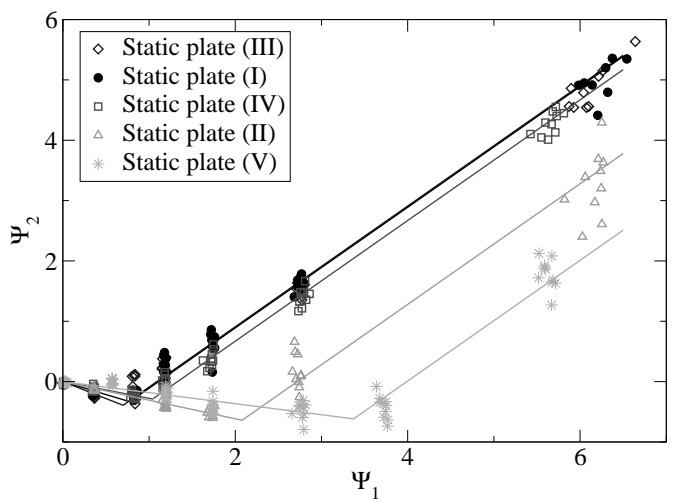

Figure 7: Collision diagram : non-dimensional local angle of contact after an impact $\psi_{2}$ as a function of the nondimensional local angle of contact before an impact $\psi_{1}$ for collisions of model particles onto five different treated static plates.

the dynamic rotating plate) using plate $(I)$. For the dynamical case, we plotted on the same graph the data for the different impacts (from the second to the fourth bounce) varying the rotating velocity of the vane from 500 to $800 \mathrm{rpm}$. This corresponds typically to a relative impact velocity varying from 1 to $7 \mathrm{~m} / \mathrm{s}$.

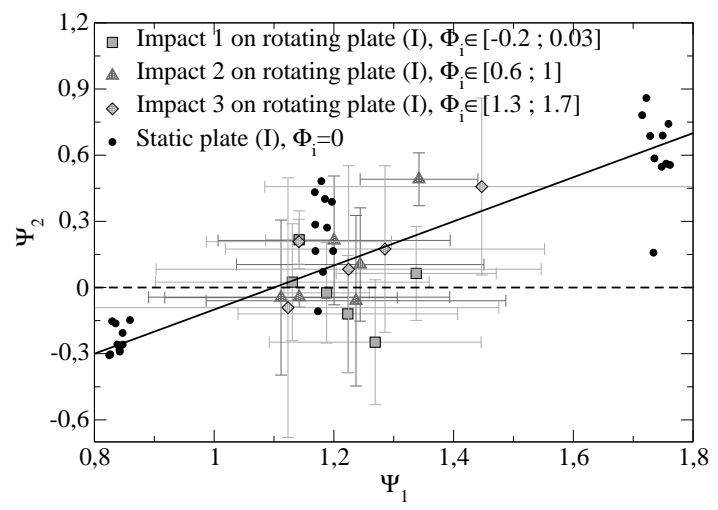

Figure 8: Collision diagram for the static case (fixed plate) and the dynamic case (rotating plate)

We reproduce again the two distinct regimes observed previously and described in [10] : (i) a first sticking regime for low values of $\Psi_{1}$ and (ii) a second sliding regime represented by a straight line for higher values of $\Psi_{1}$.

For the measurements of impact of model particles on the rotating plate, we observe that the impact angle from the normal is $0^{\circ}$ at the first impact, is $51 \pm 2^{\circ}$ at the second impact, is $64 \pm 2^{\circ}$ at the third impact and is $71 \pm 2^{\circ}$ at the fourth impact for all the experimental conditions $\left(x_{0} \in[1-8] \mathrm{cm}, \Omega \in[500-1000] \mathrm{rpm}\right.$, variation of plate material Table I). The impact angle is always more oblique than $49^{\circ}$ from the second impact. The spin of the particles has also been determined and following [6] we can define the normalized angular velocity as : $\phi_{i}=\frac{R \theta_{i}}{V_{n i}}$ where $\theta$ is the spin of the particle and 
$V_{n i}$ is the relative normal velocity on the wall. The experimental data on the collision diagram have been classified according to this parameter. This classification corresponds in fact to the order of the impacts of the particle along the rotating vane. Linear fits of the data have been done for the different values of $\phi$. For the first impact, the data are scattered and a linear fit is very rough. Indeed, the values of the spin are very small for this impact. For impact 2, we find a good interpolation by a linear curve $y=x-1,1$ and for impact 3 by a linear curve $y=x-1,08$. In the static case, the fit is $y=x-1,1$. For each impact, we note that the impact velocity varies typically from $2 \mathrm{~m} / \mathrm{s}$ to $7 \mathrm{~m} / \mathrm{s}$. According to a previous study [8], the coefficient of restitution $e_{n}$ for the experimental beads varies from the values 0,9 to 0,75 in this range of impact velocities. However, we checked that this induces only a discrepancy of $5 \%$ on the value of the friction coefficient using equation 3 . We find therefore that the difference in the data for different values of $\phi$ are not significant.

The rough correspondence between the curves for the two experimental configurations leads us to make the following comments :

(i) At first, the collisions in the experiment from the second impact appear to be in the sliding regime whatever the incident angles and the impact velocities on the plates.

(ii) Secondly the experiments show that the initial spin of the particles may be taken into account as long as the formalism of $[1,10]$ is adopted and doesn't have a clear influence on the value of the friction coefficient.

It is also important to note at this stage that a friction coefficient may be determined in a more traditional way following the seminal experiments of Coulomb [15]. Measurements of the friction coefficient with our model particles have been done by following the motion under gravity of a raft of 9 beads falling along an inclined plate for two different inclination angles $\left(\gamma=35^{\circ}\right.$ and $\left.40^{\circ}\right)$.

Experimental friction coefficients measured using 5 different treatments on the plate give the following results :

$\mu_{I}=0.38 \pm 0.02 ; \mu_{I I}=0.58 \pm 0.02 ; \mu_{I I I}=0.27 \pm 0.02 ;$ $\mu_{I V}=0.31 \pm 0.02 ; \mu_{V}=0.48 \pm 0.02$

This leads to a discrepancy ranging from 20 to $50 \%$ between both friction measurements. In order to try to explain this discrepancy, we can notice first that the normal load applied at the contact is much higher in the collision case that in the sliding case (the difference being typically equal to the inertial dynamic load $m * \frac{d V_{y}}{d t}$ where $V_{y}$ is the vertical velocity of the bead colliding the surface). If sliding friction is mainly a shearing of adhesiv junctions between microscopical asperities at the contact, then slow sliding friction occurs on a rough contact whereas impact friction may experience more plastic contacts [16]. Gorham et al(2000) [14] suggested that the derived value of $\mu$ from impact experiments must be interpreted as an effective value which takes into account some plastic deformation, unlike the measurements on inclined plates without deformation. This difference in the values of the friction coefficient might be related to the different modes of sliding between the materials [17].

\section{BOUNCING TRAJECTORY}

The measurements of the characteristic quantities for each phase of the trajectory have been done using the experimental setup presented in paragraph I. We consider also typical values $x_{0}=10 \mathrm{~cm}$ and $\Omega=100 \mathrm{rad} / \mathrm{s}$ in order to determine the dimensionless collision time scale $N_{t}$ and the centrifugal number $N_{c}$; this gives us the typical values $N_{t}=0.005$ and $N_{c}=0.0001$.

\section{A. Aerodynamic effects on the trajectory}

We checked that the air had no drag effect on the trajectory of our model particles. The typical trajectory of the particle in the rotating frame consists of a first phase of bounces with a decreasing height (cf. figure Fig. 4). We introduce the parametric equations of a bounce numbered $(i)$ without taking into account the air flow i.e. velocities of the particle in the laboratory frame $\left(V_{x i}\right.$ and $\left.V_{y i}\right)$ are supposed to be constant throughout the bounce :

$$
\begin{aligned}
x_{i}^{\prime}(t) & =X_{M} \cos (\omega)-Y_{M} \sin (\omega) \\
y_{i}^{\prime}(t) & =-X_{M} \sin (\omega)-Y_{M} \cos (\omega)
\end{aligned}
$$

We define the position of the plate as a function of the time $t: \Omega=\Omega\left(t-t_{i}\right)+\theta_{i}$ with $\theta_{i}$ and $t_{i}$ the condition at the beginning of the bounce : the orientation of the plate with the $x$-axis and the time. Supposing no air flow effect, the trajectory of the particle as a function of time is linear in the laboratory frame:

$$
\begin{array}{r}
X_{M}=x_{i}\left(t_{i}\right)+V_{x i}\left(t-t_{i}\right) \\
Y_{M}=y_{i}\left(t_{i}\right)+V_{y i}\left(t-t_{i}\right)
\end{array}
$$

With the digital video camera, we detected the initial conditions at the beginning of the bounce : $x_{i}\left(t_{i}\right), y_{i}\left(t_{i}\right)$, $\theta_{i}$ and $t_{i}$ and the positions at each step $t$ throughout the bounce $x_{i}^{\prime}(t)$ and $y_{i}^{\prime}(t)$. Fig. 9 shows the experimental data $y_{i}^{\prime}(t)$ as a function of $x_{i}^{\prime}(t)$. The line is the curve fitting of the Eqs. 5-6 with the experimental data finding the best constraint values: $V_{x i}$ and $V_{y i}$.

A maximum $3 \%$ error rate between experimental and fitted velocities $V_{x i}$ and $V_{y i}$ is observed $\left(x_{0} \in[1-8] \mathrm{cm}\right.$, $\Omega \in[500-1000] \mathrm{rpm}$, variation of plate material, Table I).

\section{B. Length of the jumping phase}

The random errors on the position of the particle are estimated as $\delta y^{\prime}= \pm 2 \mathrm{~mm}$. We consider that the particle is in a sticking phase, if the distance with the plate $y_{i}^{\prime}(t)$ 


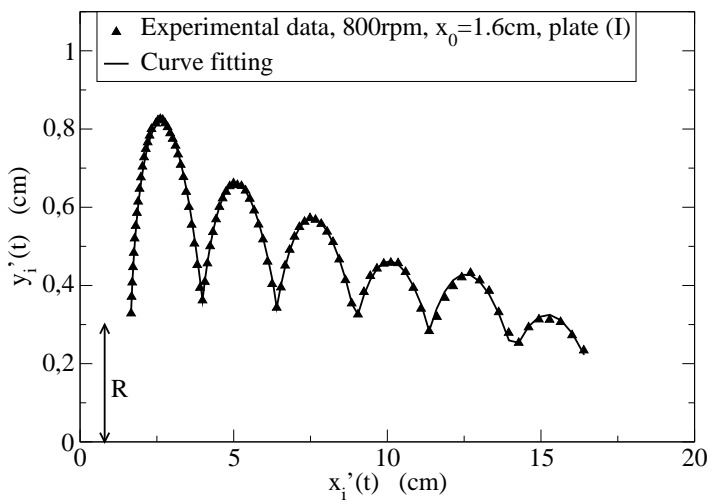

FiguRE 9: Experimental trajectory of a plastic particle onto the painted plate $\left(x_{0}=1.6 \mathrm{~cm}\right.$ and $\left.\Omega=800 \mathrm{rpm}\right)$. The line is the curve fitting of the Eqs. 5-6 with the experimental data.

is always smaller than $R+\delta y^{\prime}$. Making the hypothesis that $\tan (\Omega t) \approx \Omega t$ and the main variation of the radial velocity occurs throughout the bounces of the particle (neglecting the variation of the radial velocity during the impacts), we get the limit radial position of the bouncing phase equal to $x_{s}=\frac{x_{0}}{1-e_{n}}$ according to [13].

We give a slightly different equation than in [13] as the first impact is not treated separately. For given mechanical parameters Table I, the figure (Fig. 10) represents the experimental and theoretical evolution of the limit radial position of the bouncing phase.

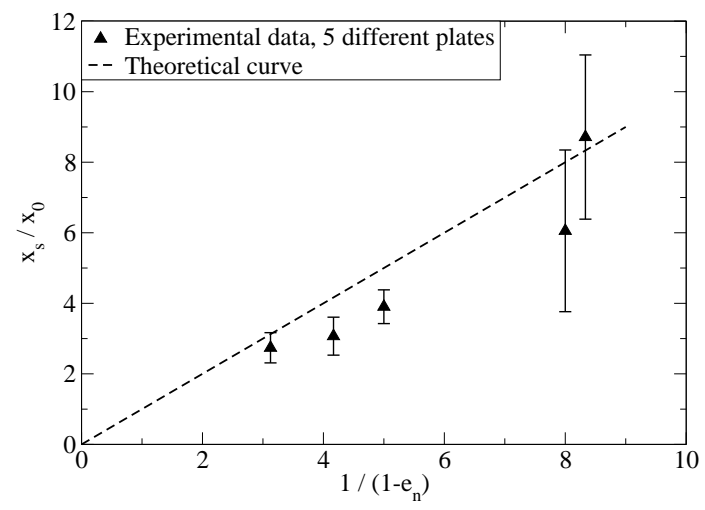

Figure 10: Experimental and theoretical limit radial positions $x_{s} / x_{0}$ as a function of $1 /\left(1-e_{n}\right)$.

A very good agreement is found between the experiments and the theory. The smaller experimental values come from the cut-off in order to determine the end of the successive bounces $\delta_{y^{\prime}}$.

\section{Sticking phase}

For positions $x>x_{s}$, the contact between the particle and the rotating plate becomes permanent. We measured the sliding velocity defined as $V_{s}=V_{x}-R \dot{\theta}$ for each experimental conditions $\left(x_{0} \in[1-8] \mathrm{cm}, \Omega \in[500-1000] \mathrm{rpm}\right)$.
The particle is supposed to be in a rolling without sliding regime if the sliding velocity measurement is smaller than the random errors $\delta V_{s}=\delta V_{x}+\delta R \dot{\theta}$. Figs. 1112 represent the sliding velocity along the vane with the particle onto the painted and roughen steel plate $\left(x_{0}=1.6 \mathrm{~cm}, \Omega \in[500-800] \mathrm{rpm}\right)$.

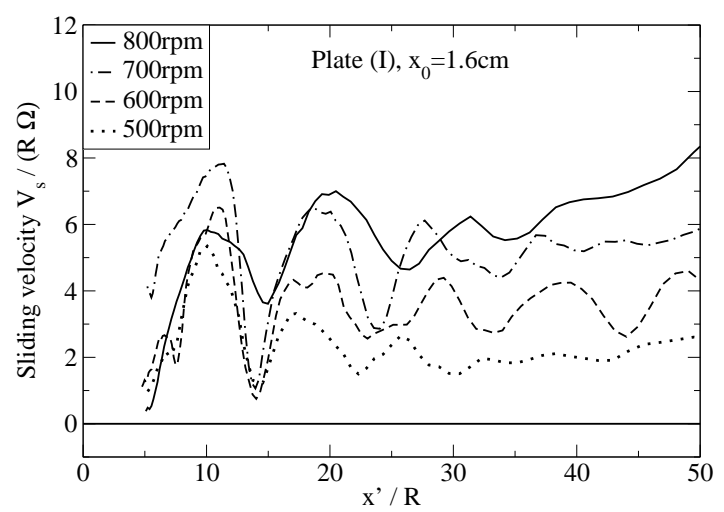

FiguRE 11: Dimensionless sliding velocity along plate (I) $\left(x_{0}=1.6 \mathrm{~cm}\right)$.

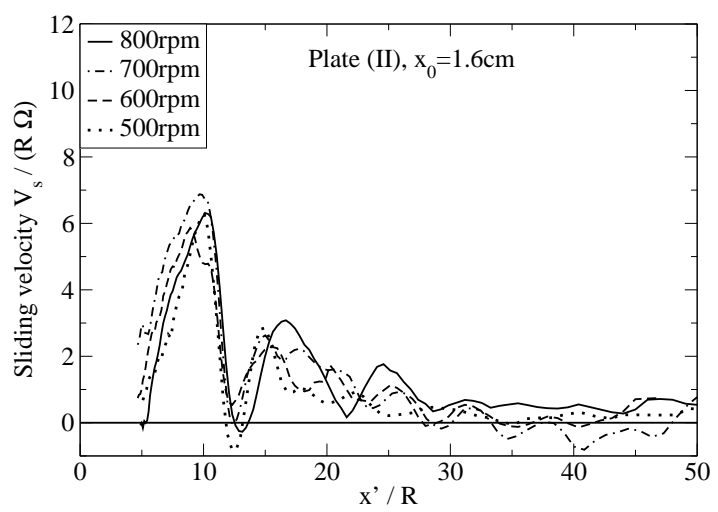

FigurE 12: Dimensionless sliding velocity along plate (II) $\left(x_{0}=1.6 \mathrm{~cm}\right)$.

We observe clearly the appearance of two distinct regimes. The sliding velocity is always greater with plate (I) $(\mu=0.175)$. With the roughen plate (II) $(\mu=0.45)$, the sliding velocity is reaching zero. The particle on plate $(I)$ is supposed to be in a rolling with sliding regime $(R+S)$, whereas the particle on plate $(I I)$ is supposed to be in a rolling without sliding regime $(R-S)$. The sliding velocity depends also on the rotation velocity $\Omega$.

In order to estimate the value of the critical friction coefficient $\mu^{\star}$, Fig. 13 represents the sliding velocity using five different treated materials at a rotating velocity of $\Omega=700 \mathrm{rpm} .\left(\delta V_{s} / R \Omega=2.5\right)$.

For all plate materials, the sticking phase appears at $x^{\prime}=x_{0} /\left(1-\max \left(e_{n}\right)\right) \approx 13 \mathrm{~cm}$. We observe that the sliding velocity decreases with the friction coefficient. The sliding velocity is only greater than the random errors with the painted (plate I) and the varnished (plate III) 


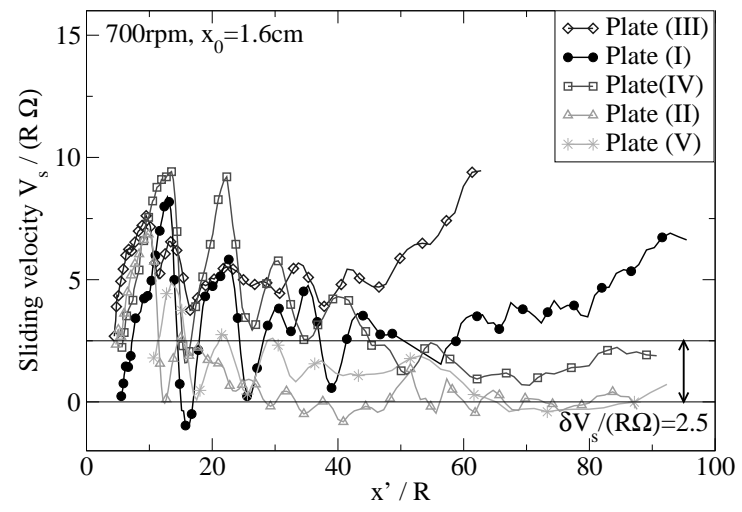

Figure 13: Dimensionless sliding velocity along the different plate materials $\left(x_{0}=1.6 \mathrm{~cm}, \Omega=700 \mathrm{rpm}\right)$

steel plates. From these data, we can give a frame of the value for the critical friction coefficient : $\mu_{I} \leq \mu_{e x p}^{*} \leq \mu_{I V}$ with $\mu_{I}=0.175 \pm 0.005$ and $\mu_{I V}=0.205 \pm 0.003$. Varying the velocity of the plate between 500 rpm. and 800 rpm., we notice that the same estimation of the critical friction coefficient holds in conformity with the theory proposed recently [13].

We note also that all the combinations of particle and plate material (Table I) have a fairly high value of normal restitution coefficient : $e_{n} \in[0.68-0.88]$. Hence the critical friction coefficient estimated experimentally is in very good agreement with the theoretical value predicted for high normal coefficients of restitution $(\approx 0.169)$.

The rotational speed at the limit radial position $x_{s}$ for a particle in a $(R+S)$ regime is :

$$
R \dot{\theta}_{s}=R \dot{\theta}_{1}+\frac{5}{2} x_{0} \Omega \mu e_{n} \frac{1+e_{n}}{1-e_{n}}
$$

In the same way, with all the particles in a $R-S$ regime, $\mu=\mu^{\star}=1 / \sqrt{35}$ and $R \dot{\theta}_{s}(\mu)$ is a saturated function of $\mu$. Throughout the first bounce, a small negative rotational speed $R \dot{\theta}_{1}$ is observed, a feature which is not taken into account in the theory $\left(R \dot{\theta}_{1}=\frac{5}{2} x_{0} \Omega \mu\left(1+e_{n}\right)>0\right.$ according to the theory). The existence of an initial angular velocity $R \dot{\theta}_{1}$ can be explained by an effect of the tangential compliance since a small radial velocity $V_{x 1}^{\prime}$ is also observed just after the first impact. The first impact angle occurs always at an impact angle of $0^{\circ}$ from the normal. In these range of values, the tangential compliance is expected to give rise to specific features for the impact such as a negative rebound angle [14].

Fig. 14 shows the experimental rotational speed at the end of the bouncing phase. The dotted line is the curve fitting of the Eq. 7 with the experimental data finding the best constraint value : $R \dot{\theta}_{1}$. A good estimation for the rotational speed is $R \dot{\theta}_{s}=-0.26 x_{0} \Omega+\frac{5}{2} x_{0} \Omega \mu e_{n} \frac{1+e_{n}}{1-e_{n}}$.

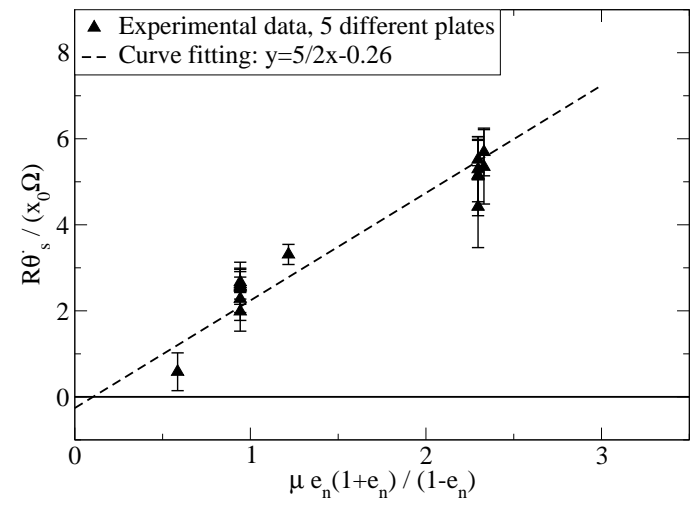

Figure 14: Experimental and theoretical rotational speed at the end of the bouncing phase.

\section{Conclusion}

We presented in this article new results concerning the impact of spinning particles along moving boundaries for different surface properties. We showed experimentally that the bouncing trajectory consists in a succession of rebounds of decreasing height followed by a permanent contact of the particle along the vane. These dynamics can be reproduced by the simple theory proposed recently [13] in the limit where the centrifugal number and the non dimensional collision time are small compared to one. The condition for this matching is that the normal restitution coefficient and the friction parameter have to be measured independantly using an experimental device of impact of a falling bead on a static plate according to [10]. A consequence of this study is that the effect of the initial spin of the particle before each impact can be taken into account by using an apparent impact angle defined by the normal and tangential velocity components of the particle surface as explained recently [6]. This is in fact implicit in the formulation of [10] for the study of the impact. Also, the tangential restitution coefficient characterizing the tangential compliance doesn't need to be taken into account according to our previous theoretical treatment [13] : from the second impact onto the rotating plate, the impact angle is indeed oblique enough to be accurately described by a theory of frictional impact in agreement with [14]. For values of the friction coefficient higher than a critical value, we observe that the sliding velocity of the particle along the vane cancels and that the particle reaches a rolling without sliding regime $(R-S)$ in agreement with the theory. The model is able to predict quantitatively the critical value of this friction coefficient. Another interesting feature of the experiments is the observed difference in the values of the friction coefficients particle/plate measured from the two different protocols used (impact on a static plate - Coulomb friction experiment) at a low velocity (1 to $2 \mathrm{~m} / \mathrm{s}$ ). In fact, at the scale of the contact, the apparent friction coefficient may involve surfacic properties of the materials as well as volumic properties such that its 
value may depend on the precise stress path applied at the interface between the two bodies [16]. This may have some consequences in the determination of the boundary conditions for frictional collisional granular flows because particles may not experience the same friction along the boundary depending on their specific motion (impact or sliding). A deeper analysis of the difference between these two quantities at the scale of the contact will be of much value.

Another possible perpective of this study is to extend the experiments to other kinds of softer particles and surfaces. For the last case, new properties for the impact may be expected as the centrifugal number $N_{c}$ and the nondimensional collision time $N_{t}$ might not be small compared to 1 [13]. The influence of the asphericity of the bead has also to be considered. We already observed unexpected bouncing trajectories for angular particles on some surfaces in the present setup.

\section{Acknowledgments}

We would like to acknowledge a financial support from the région Auvergne (contributing to the PHD grant of A. Le Quiniou). This work also benefitted from the support of the Fédération de Recherche TIMS, CNRS 2856. C.Caroli and T.Baumberger are aknowledged for fruitful discussions concerning the physics of solid friction, M.Y.Louge, C.Thornton, T.Biben, H.H.Shen for their comments about the models of impact and B.Elhedili (an IFMA student) for interesting suggestions about this work.
[1] N. Maw and J. R. Barber and J. N. Fawcett, "The oblique impact of elastic spheres", Wear, 38, No.l,pp 101-114, 1976

[2] , O.R.Walton, "Granular Solids Flow Project," Quarterly Report N. UCID-20297-88-1, Lawrence Livermore National Laboratory, 1988

[3] , K.L. Johnson, "Contact mechanics", C.U.P. 11, 1985

[4] , W. J. Stronge and R. James and B. Ravani, "Oblique impact with friction and tangential compliance", Philosophical Transactions of the Royal Society London A, 359, 2447-2465, 2001

[5] H. Dong and M.H. Moys, "Experimental study of oblique impacts with initial spin", Powder Tech., 161, 22-31, 2006

[6] C. Thornton, "A note on the effect of initial particle spin on the rebound behaviour of oblique particle impacts" Powder Tech., 192, 152-156, 2009

[7] E. Falcon and C. Laroche and S. Fauve and C. Coste, "Behavior of one inelastic ball bouncing repeatedly off the ground", Eur. Phys. J. B, 5, 111, 1998

[8] F. Rioual and A. Le Quiniou and Y. Lapusta, "impact of a particle along a rotating wall", Comptes rendus de mécanique, 336, p.664-669, 2008

[9] P. Van Liedekerke and E. Tijskens and H. Ramon, Pow- der Tech.," Discrete element simulations of the influence of fertiliser physical properties on the spread pattern from spinning disc spreaders", 102, 392-405, 2009

[10] S. F. Foerster and M. Y. Louge and H. Chang and K. Allia, "Measurements of the collision properties of Small Spheres", Phys. Fluids, 6(3), 1108-1115, 1994

[11] R. Sondergaard and K. Chaney and C. E. Brennen, "Measurements of solid spheres bouncing off flat plates", J. Appl. Mech., 57(3), 694-699, 1990

[12] C. Zener, "The intrinsic elasticity of large plates", Phys. Rev., 59, 669-673, 1941

[13] A. Le Quiniou and F. Rioual, "Flow of a particle along a rotating wall", Europhys. Lett., 82, 34001, 2008

[14] D. A Gorham and A. H. Kharaz, "An experimental study of the elastic rebound of spheres", Powder Tech., 112, 193-202, 2000

[15] C.A. Coulomb, "Théorie des Machines simples", d. Blanchard, Paris (2002)

[16] F.P.Bowden and D.Tabor, "The friction and lubrication of solids", Clarendon Press, Oxford, 1986

[17] R. M. Brach,"Impact dynamics with application to solid particle erosion", Int. J. Impact Engng., 7, 37-53, 1988 


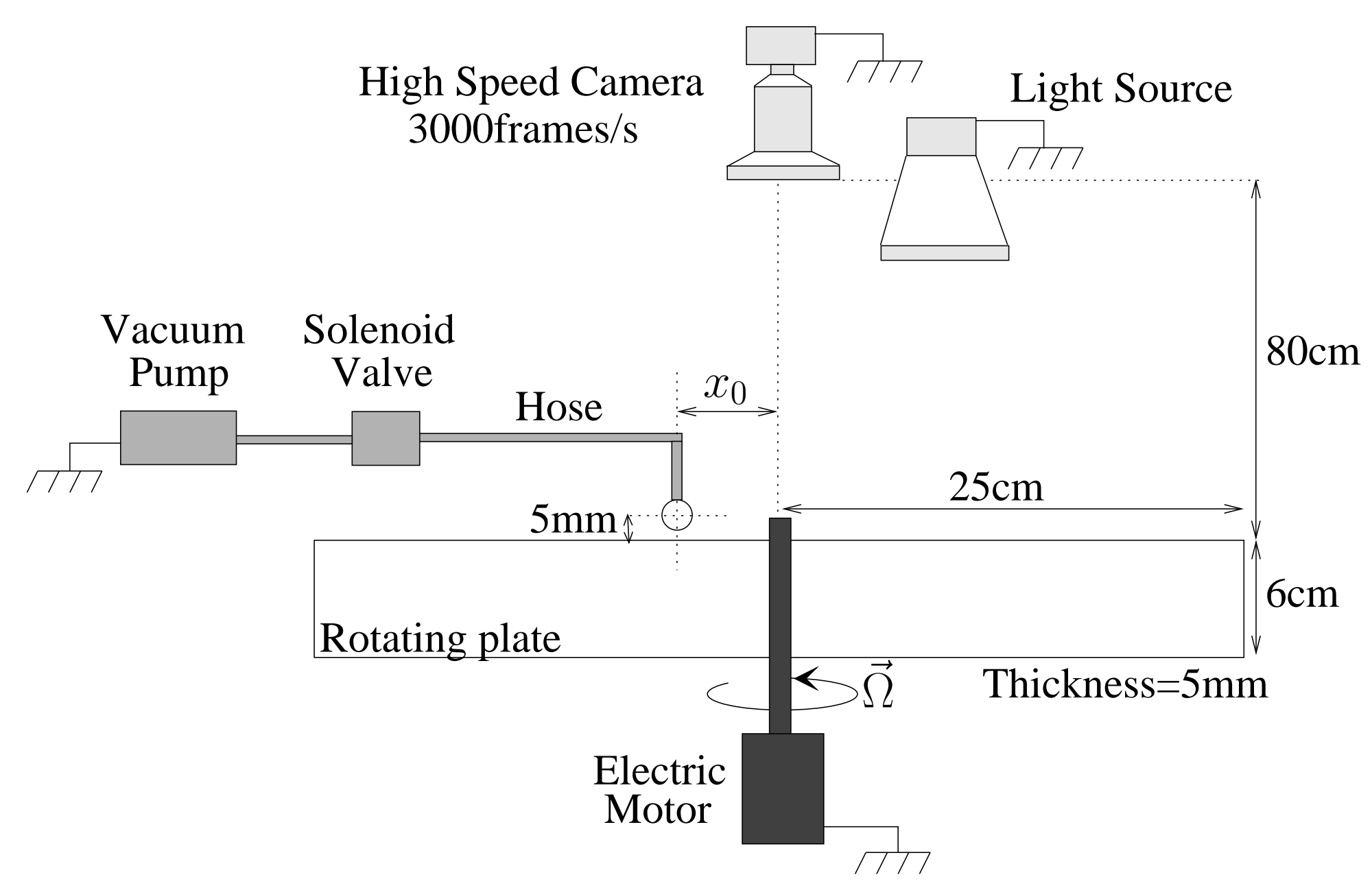




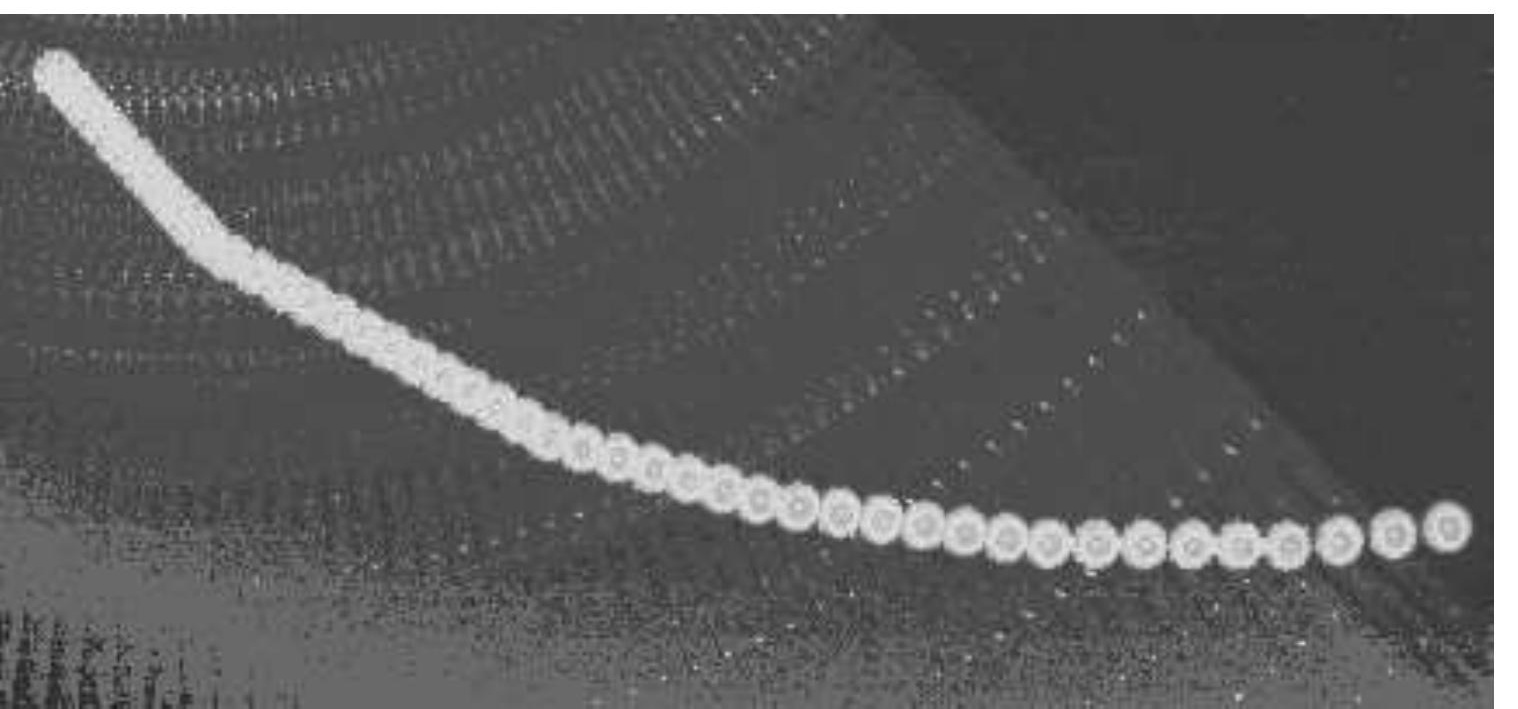




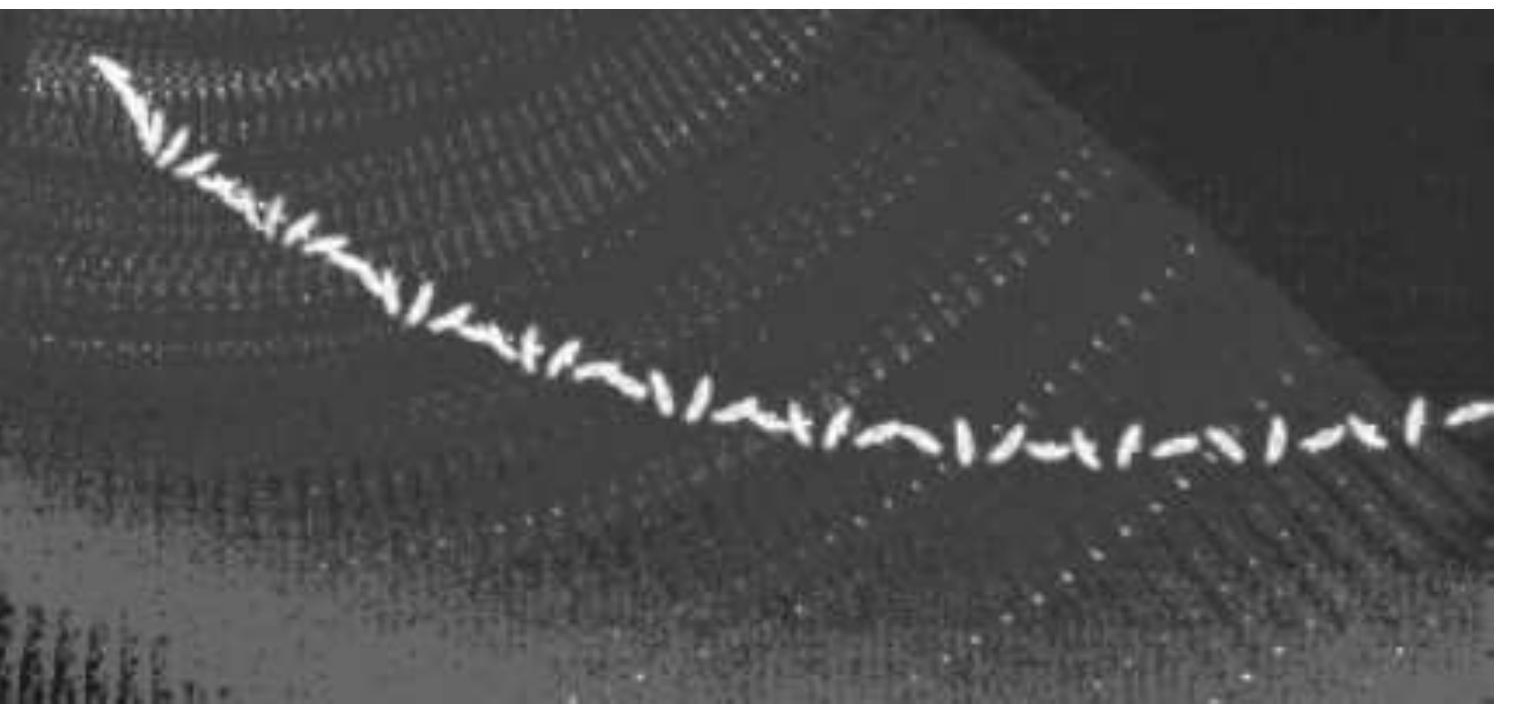




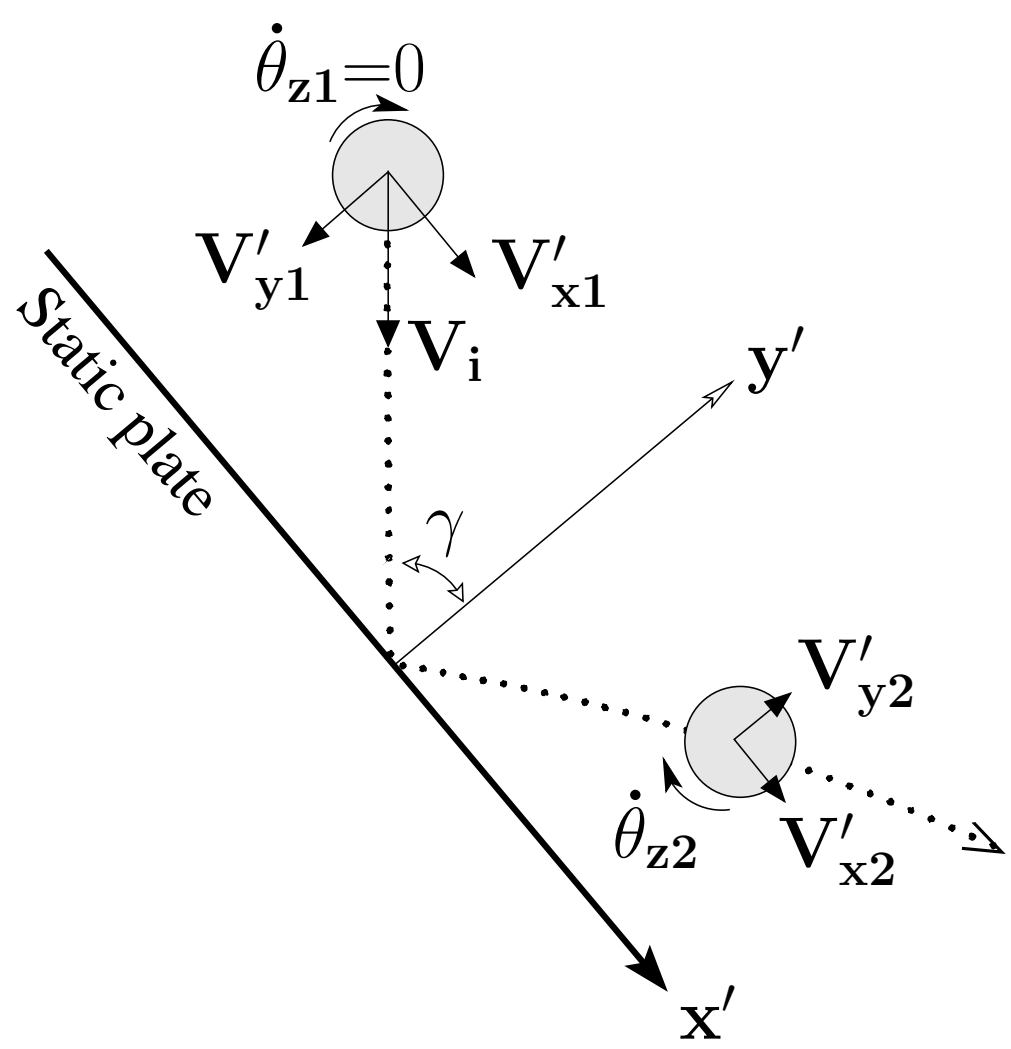




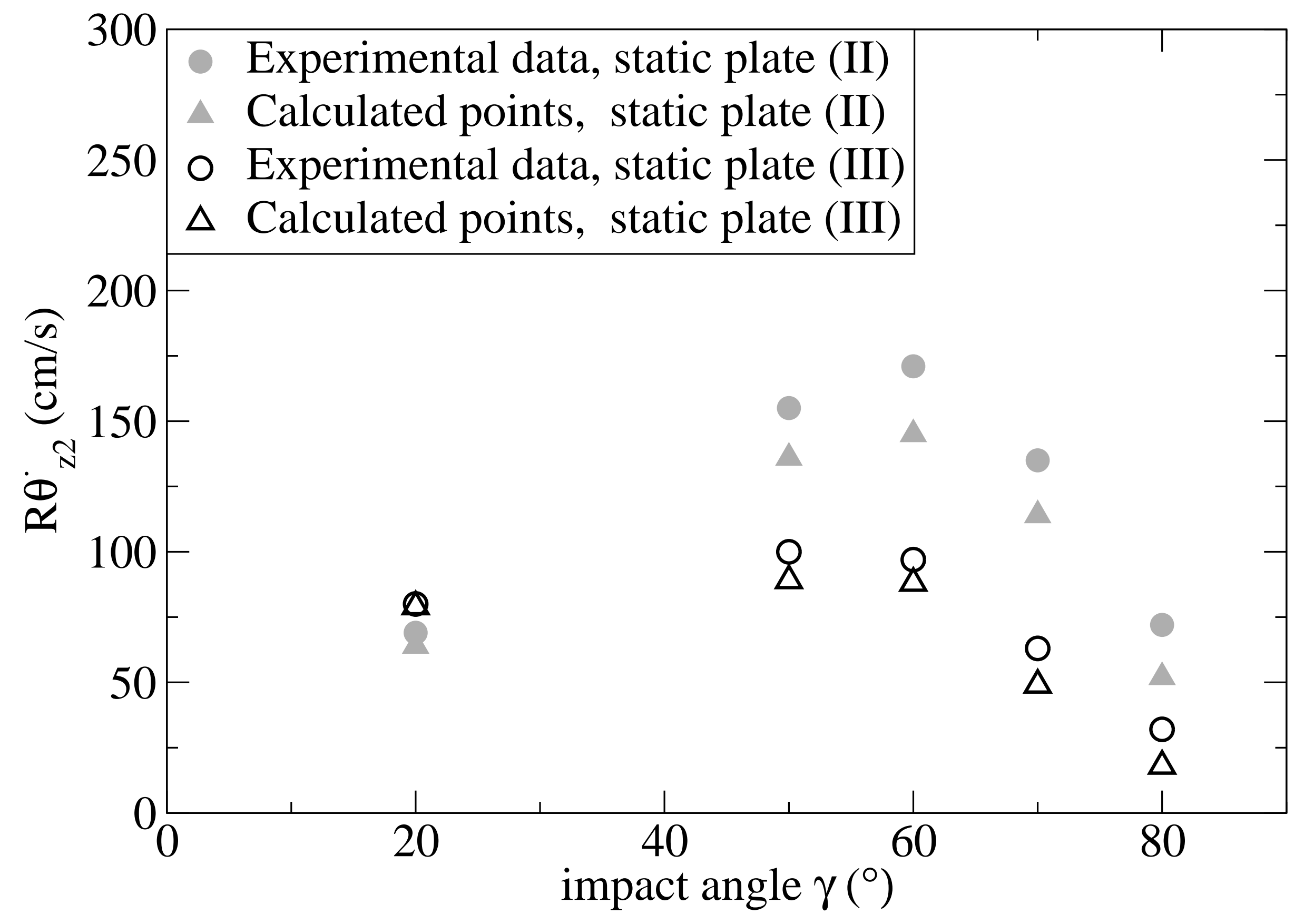




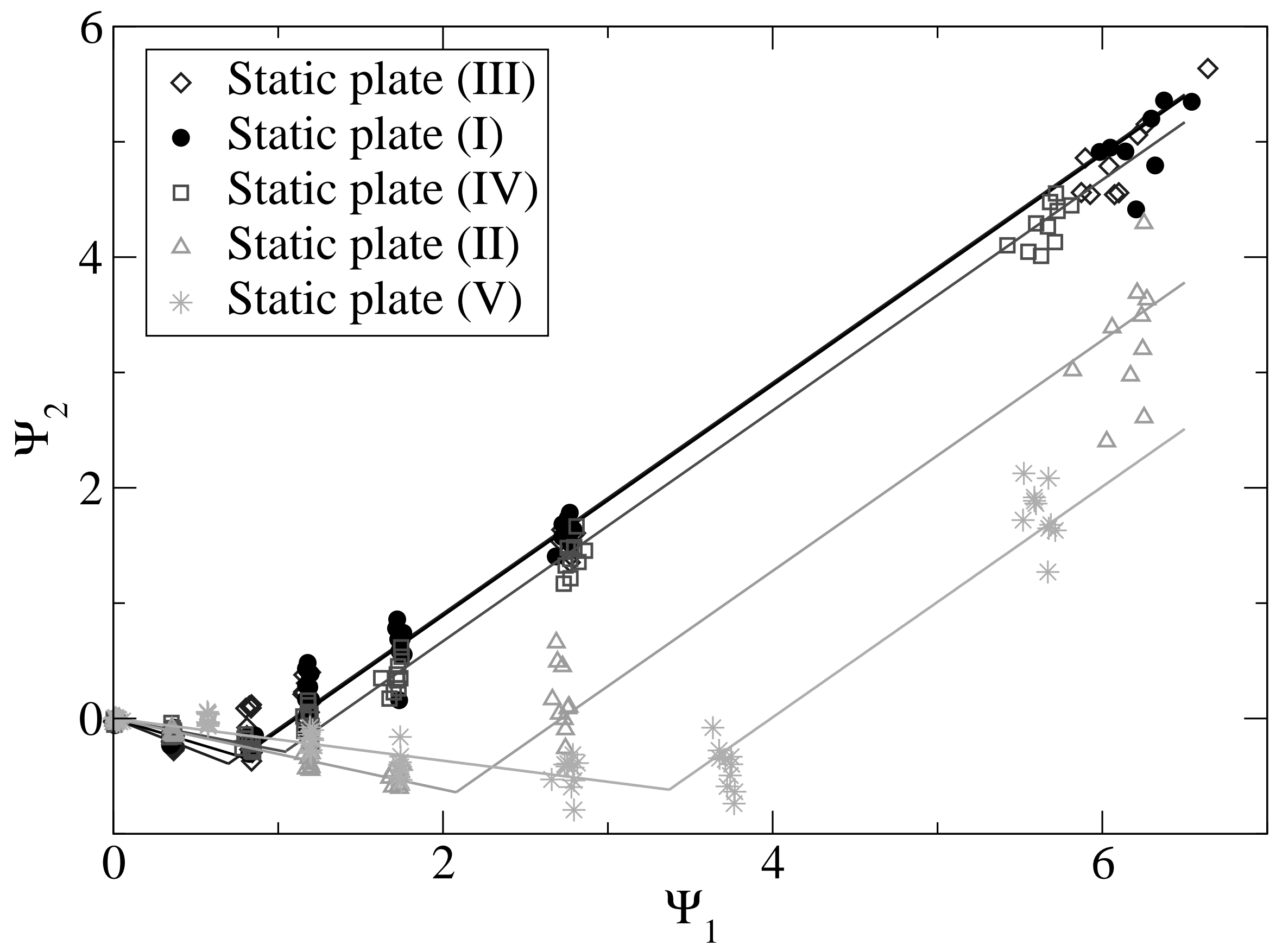




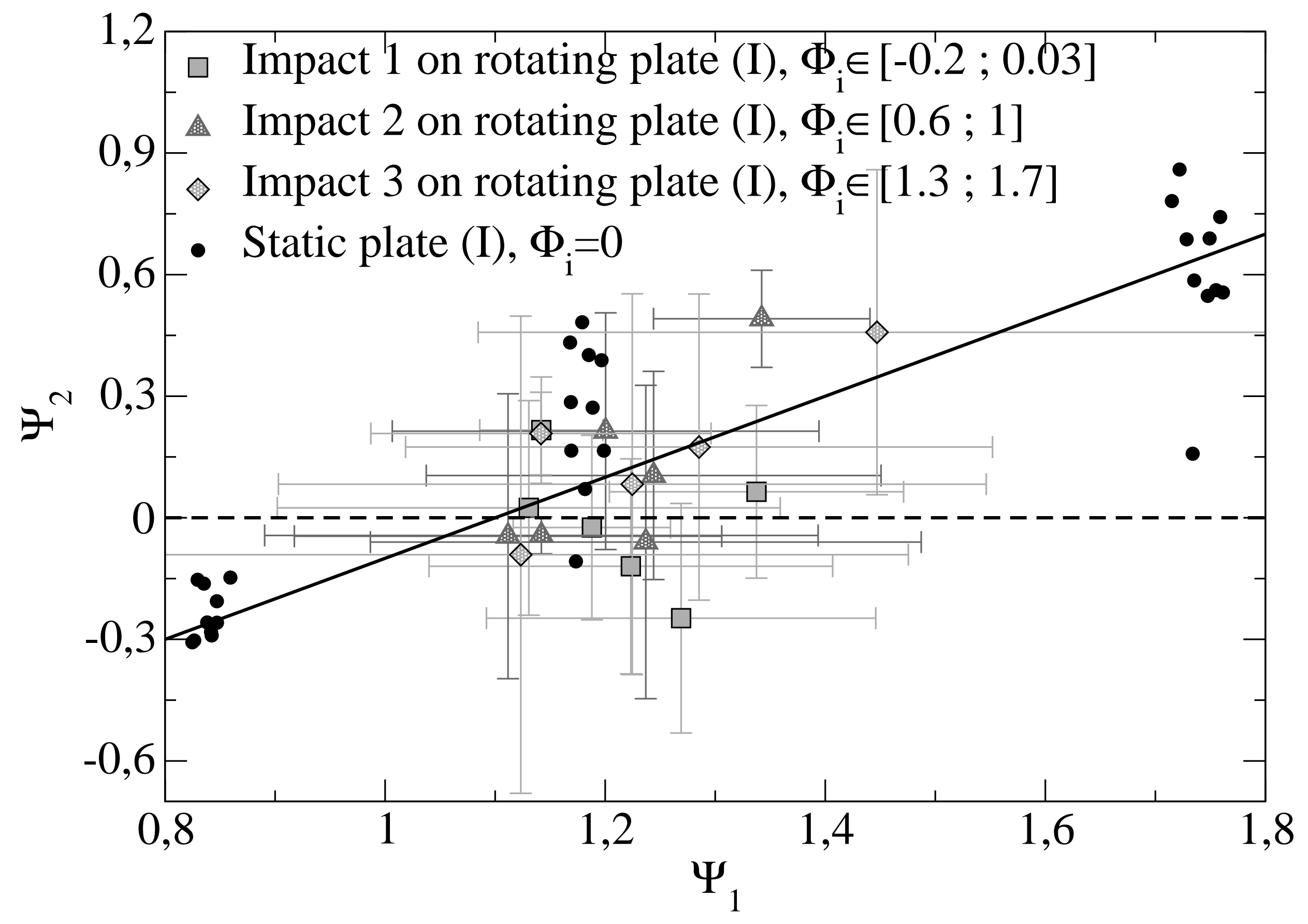




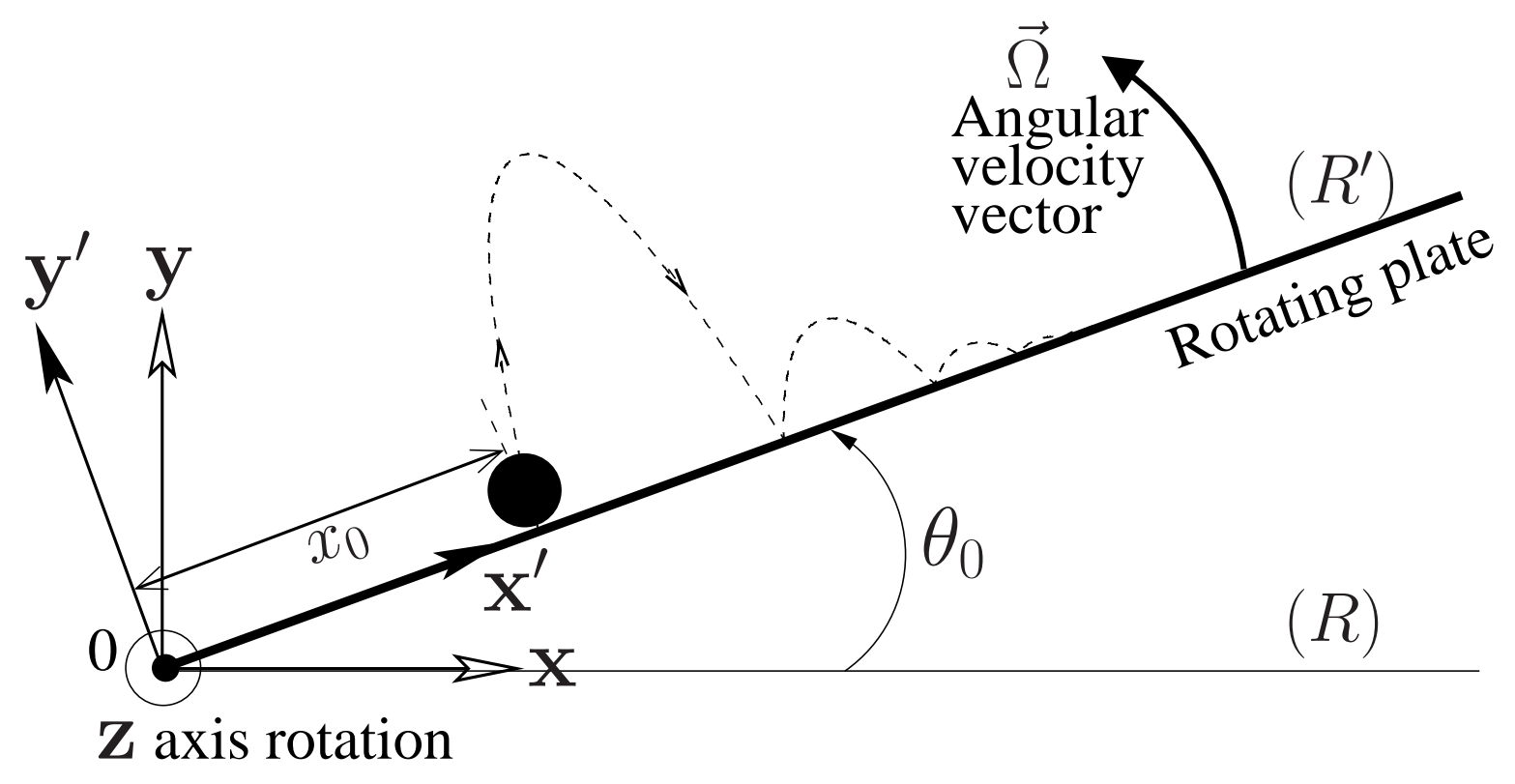




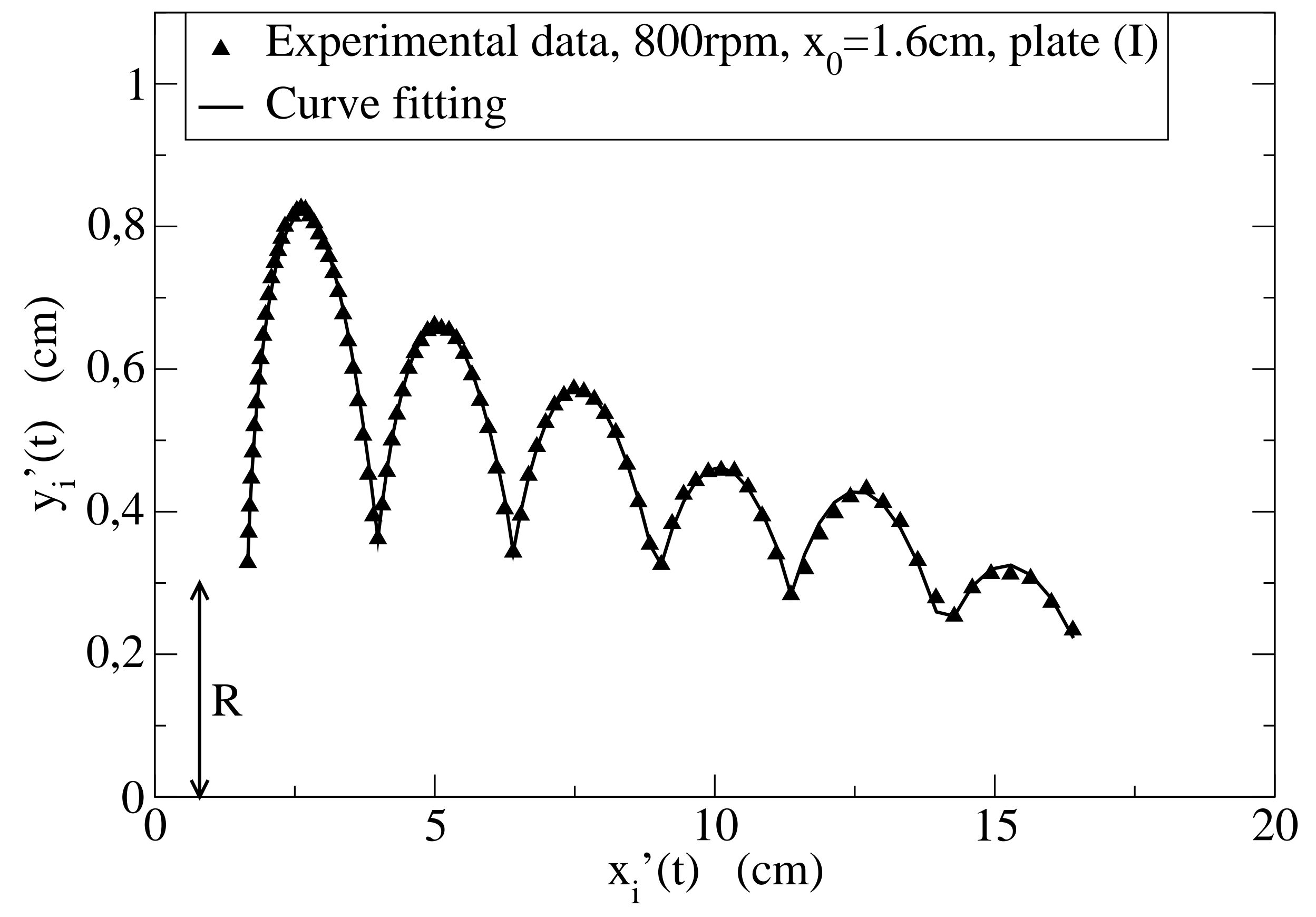




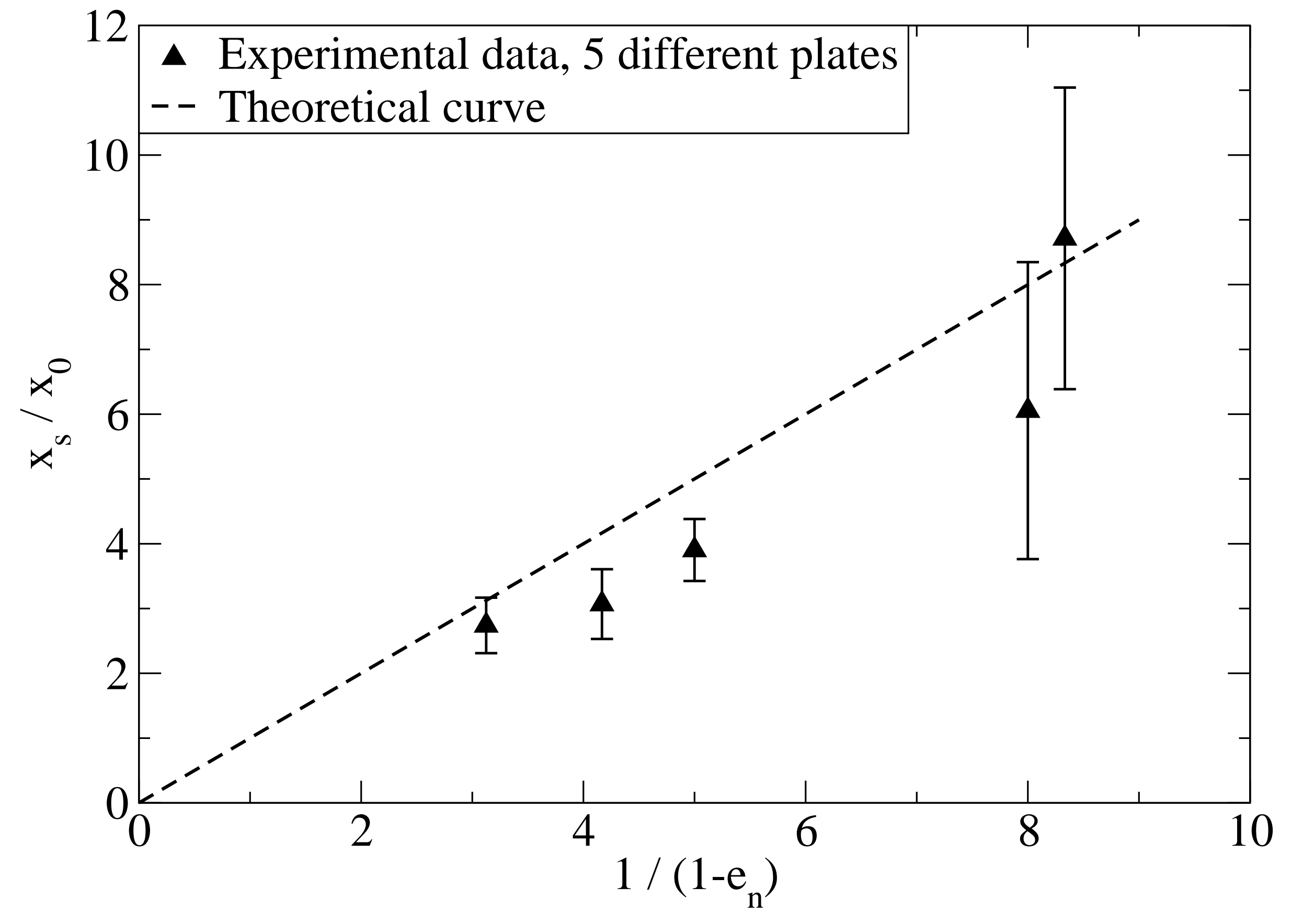




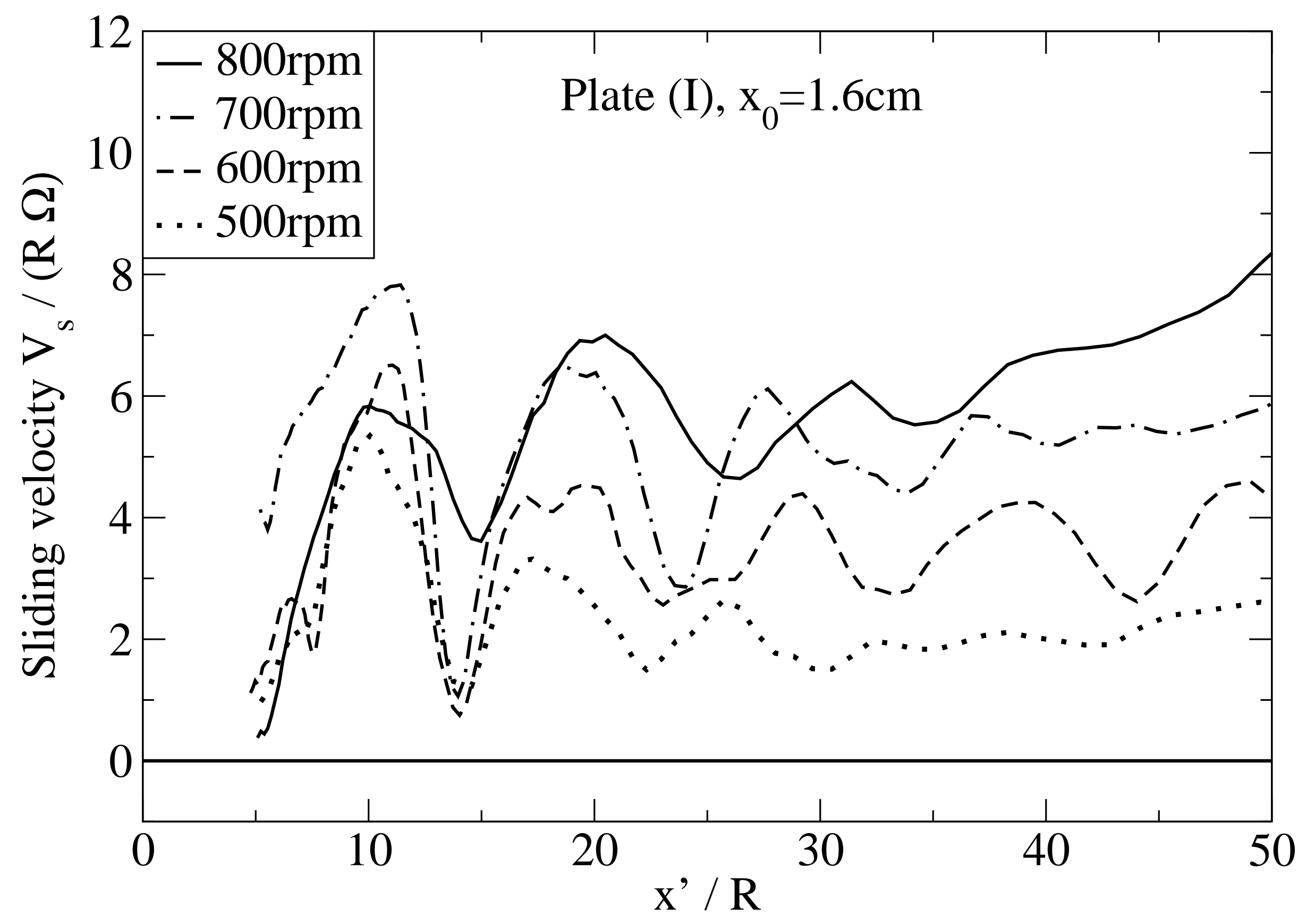




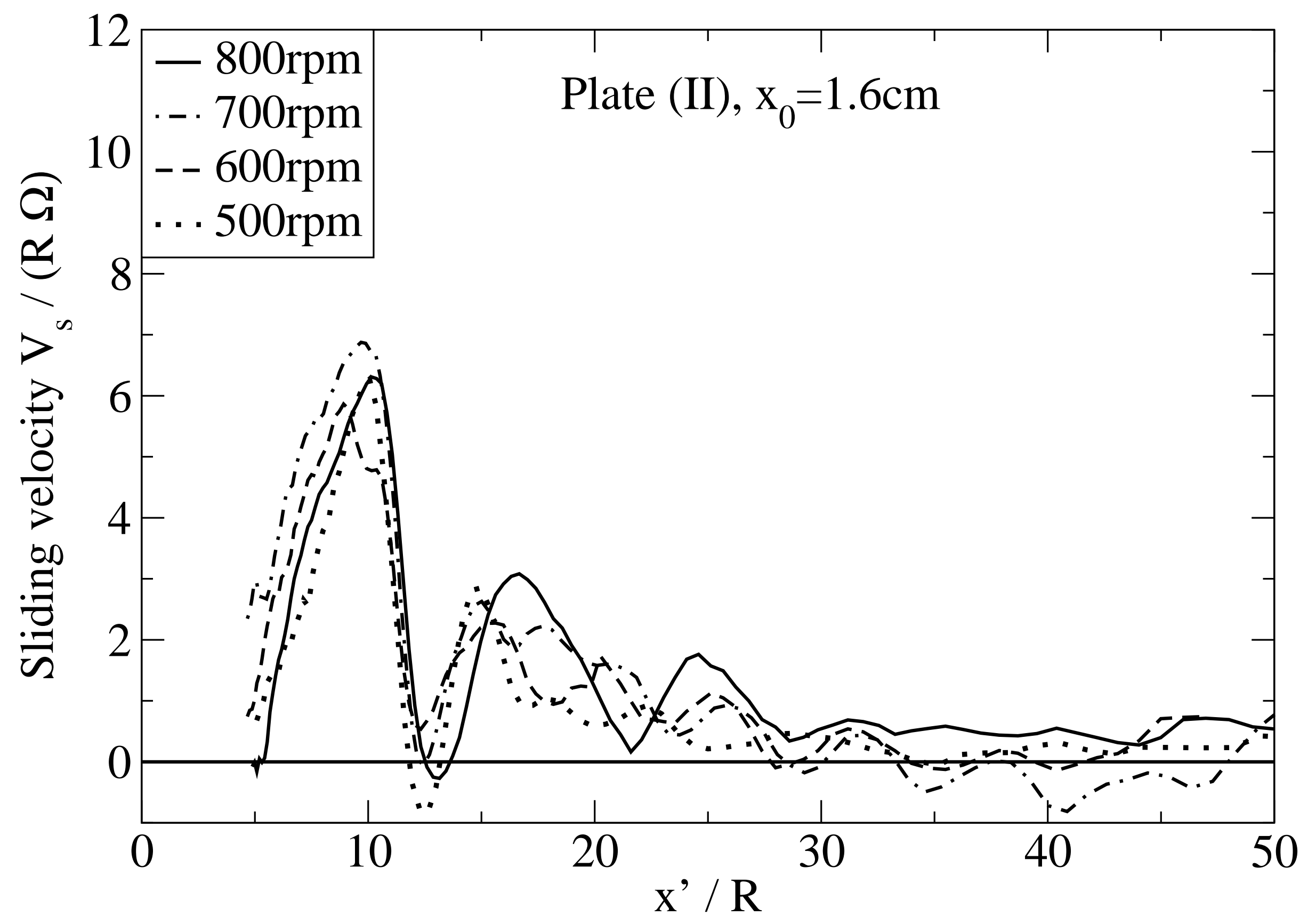




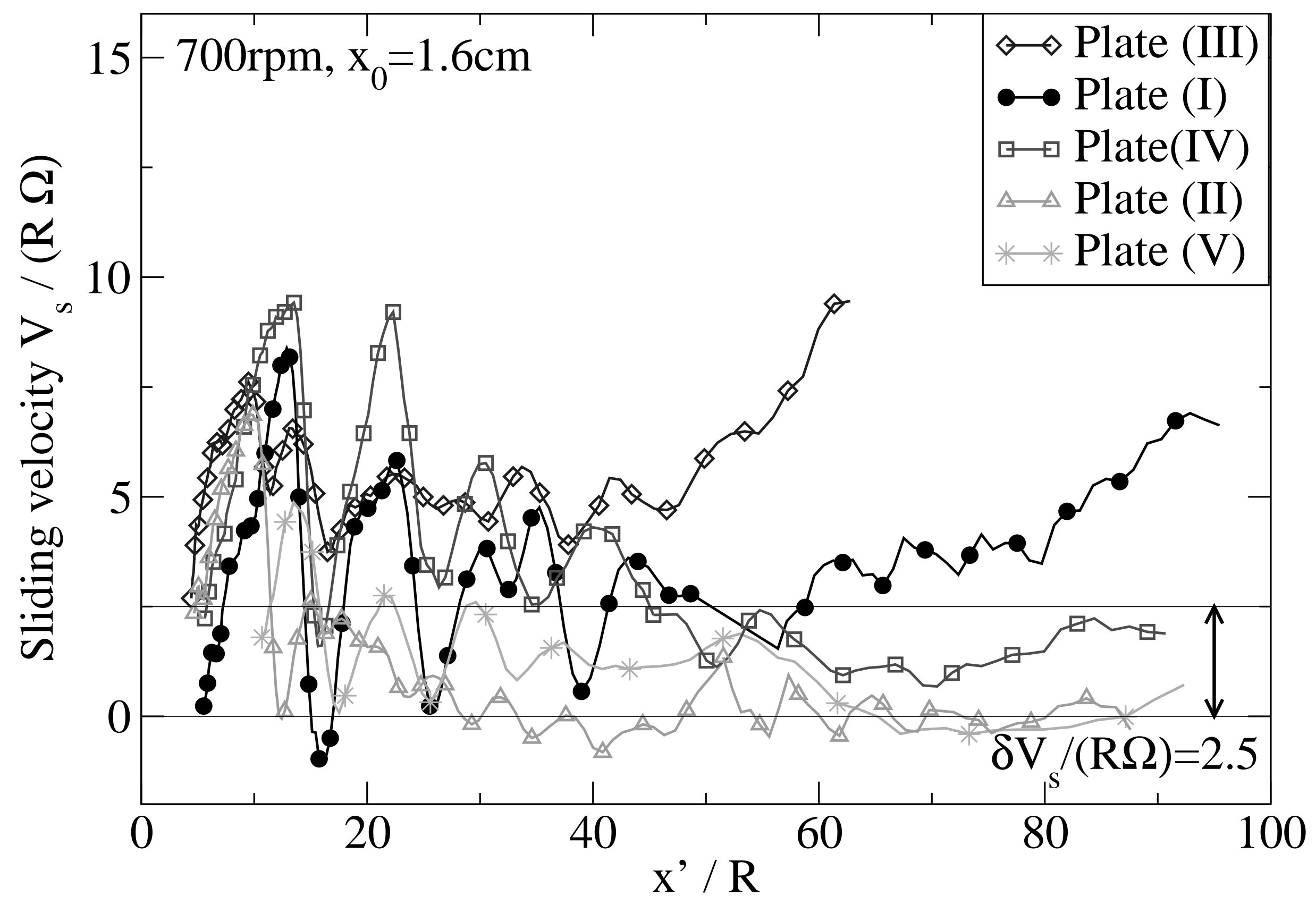




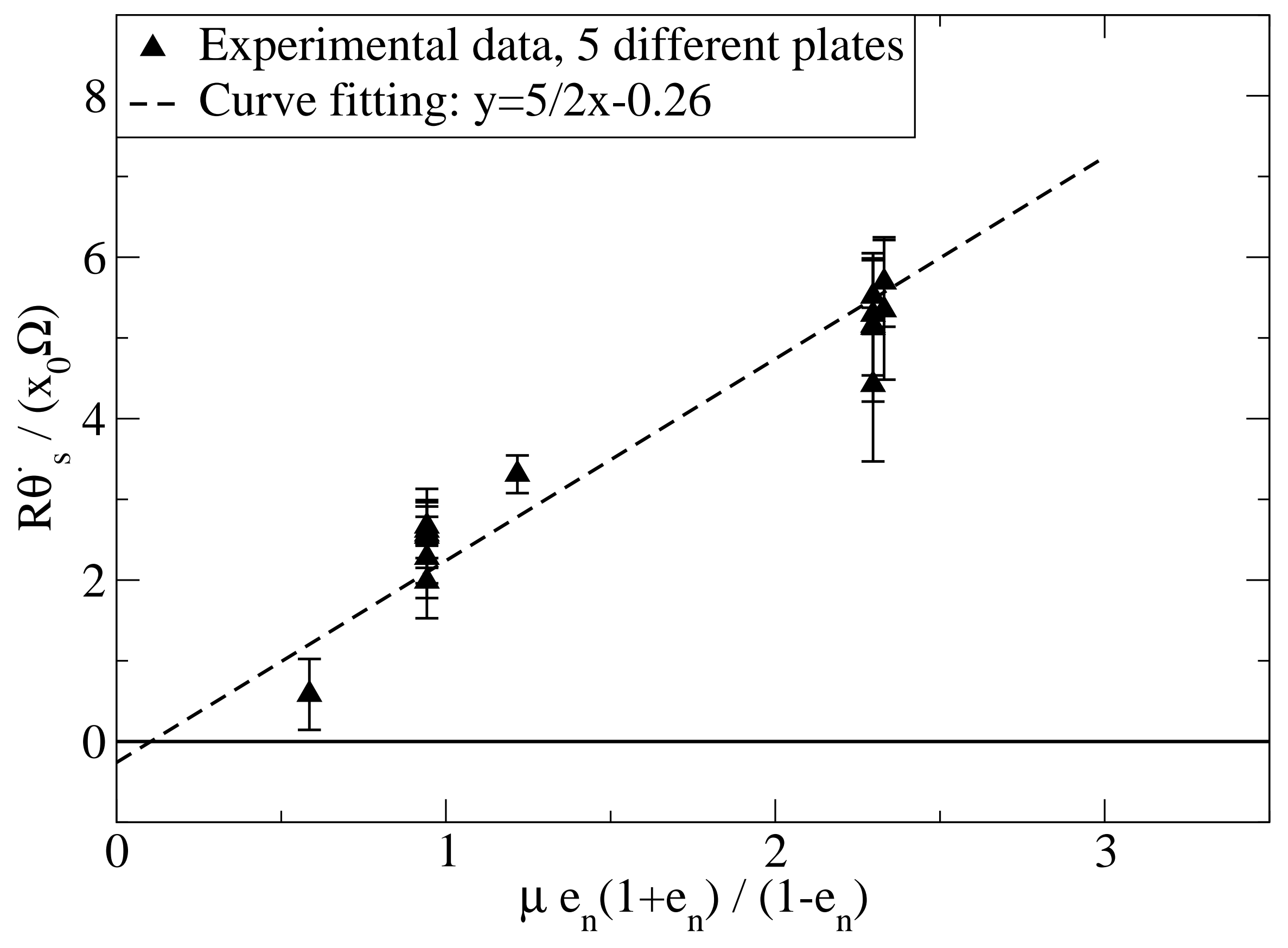

